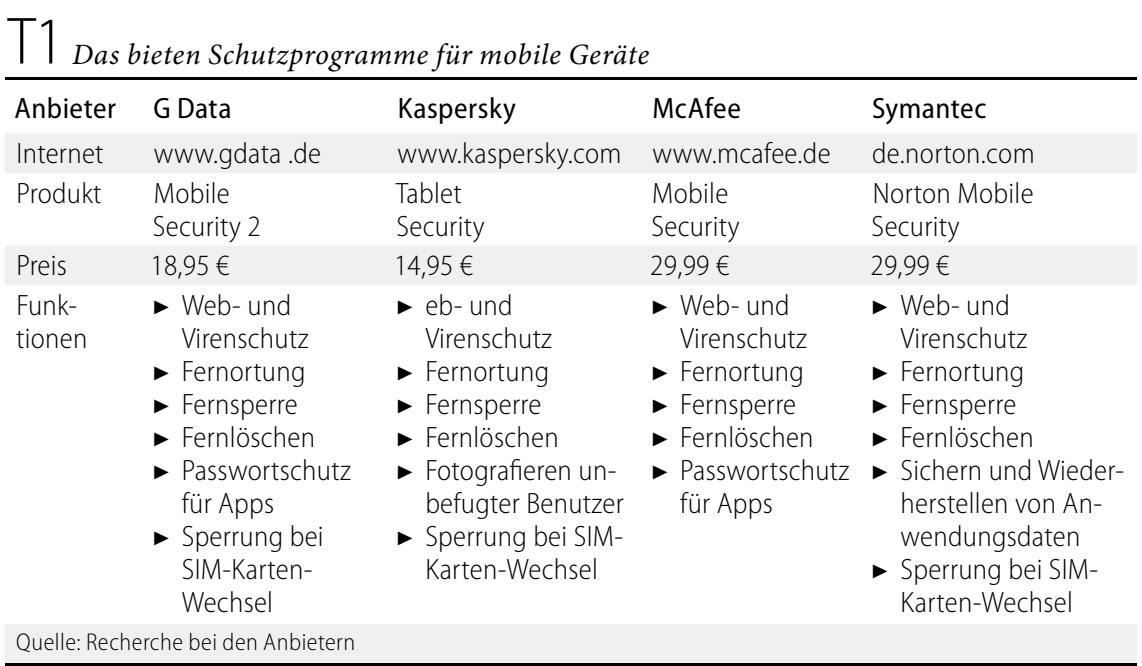

Gewinn: Der Markt für ausgespähte Kreditkartendaten und Zugänge zu Online-Banking oder Online-ShoppingSystemen ist deutlich größer als der Handel mit Patientendaten oder digitalen Untersuchungsergebnissen. Doch zum einen können solche sensiblen $\mathrm{Da}$ ten als Beifang die gesicherte Praxisumgebung verlassen, was per se schon einen Datenschutz-GAU darstellt. Zum anderen bieten die empfindlichen Informationen durchaus Möglichkeiten zur Monetarisierung durch Datendiebe - man denke etwa an Erpressungsversuche oder den Weiterverkauf von Patientendaten an zwielichtige Adresshändler. Benzmüller: „Viele Computernutzer unterschätzen den Wert ihrer Daten.“

\section{Niemals ohne Sperrfunktion arbeiten}

Es liegt auf der Hand, auch die mitgebrachten Geräte müssen abgesichert werden. Neben den auf Einzelanwender zugeschnittenen Schutz-Apps beziehen Sicherheitslösungen für Unternehmen und Institutionen mobile Geräte längst in ihre Schutzkonzepte mit ein ( Tabelle 1). Bereits bei der Installation neuer Apps überprüfen die Sicherheitsprogramme, ob es sich um einen Schadcode handelt. So wird die Ausführung von Spionage- oder Betrugsfunktionen vor deren Aktivierung verhindert und das Programm nicht installiert. Zeitgemäße Sicherheitsprogramme gehen noch einen Schritt weiter und haben auch bei regulären Apps die Rechte im Blick. So haben Datenkraken keine Chance und Anwender wissen jederzeit genau, welche Anwendungen welche
Berechtigungen umfassen, zum Beispiel den Zugriff auf das Adressbuch oder auf das Internet.

Ein weiteres Szenario, das Schutzprogramme für mobile Endgeräte abdecken, ist der Verlust des Geräts. Dabei geht es nicht nur um die Ortung via eingebauter GPS-Funktion. Noch wichtiger ist es, ein abhandengekommenes Gerät aus der Ferne sperren oder bei Bedarf sogar komplett löschen zu können, damit ein Dieb oder zufälliger Finder keinen Zugriff auf die darauf gespeicherten sensiblen Daten erhalten kann.

Wer mit sensiblen Daten zu tun hat, sollte keinesfalls auf ein leistungsstarkes und technologisch aktuelles Sicherheitsprogramm auch auf mobilen Geräten verzichten. Hinzu komme, so Sicherheitsexperte Benzmüller immer auch noch der Faktor Mensch: „Eine wichtige Dimension in jedem Schutzkonzept ist verantwortungsvolles und sicherheitsbewusstes Verhalten der Anwender beziehungsweise Mitarbeiter". Patientendaten und Untersuchungsergebnisse sollten nicht dauerhaft auf dem privaten Gerät gespeichert bleiben. Auf Endgeräten, die in sensiblen Bereichen zum Einsatz kommen, muss vielleicht auch nicht unbedingt "Angry Birds“ gespielt werden. Und bei vermeintlichen Gratisangeboten oder verdächtigen E-Mails ist ein vernünftiges $\mathrm{Maß}$ an Skepsis angebracht. "Wer solche simplen Verhaltensregeln befolgt, und dann noch ein geeignetes Schutzprogramm nutzt, ist in puncto Sicherheit schon einen großen Schritt weiter." Hannes Rügheimer

\title{
One-Click-Abrechnung seit Juli in allen PVS verfügbar
}

Seit dem 1. Juli ist es verbindlich: $\mathrm{Ab}$ dem dritten Quartal müssen alle von der KBV zugelassenen Anbieter von Praxisverwaltungssystemen (PVS) den Kunden die „One-Click-Abrechnung“ erlauben. Die Funktion soll den Praxen ermöglichen, ihre Quartalsabrechnung elektronisch direkt an ihre zuständige $\mathrm{KV}$ zu senden. Als Übertragungsweg dient laut KBV der Kommunikationskanal KV-Connect, der von der Telematik-Arbeitsgemeinschaft der KVen entwickelt wurde. Alternative Arten der Online-Abrechnung werden weiterhin von der KV akzeptiert. Rebekka Höhl

\section{Jeder Zweite nimmt seinen Tablet-PC mit in das Bett}

Sie sind handlich und mobil: Tablet Computer. Wie sie genutzt werden, zeigt eine repräsentative Befragung von Bitkom Research. Demnach setzt jeder vierte Nutzer seinen Tablet ausschließlich und weitere $34 \%$ überwiegend $\mathrm{Zu}$ hause ein. $30 \%$ verwenden ihren Tablet gleichermaßen dort und unterwegs. Dabei nimmt fast jeder Zweite seinen Tablet mit in das Bett, bei weiteren $34 \%$ ist er am Küchentisch - etwa als Kochbuch - in Gebrauch. 92\% der 509 befragten Tablet-User sitzen damit auf dem Sofa. Am Schreibtisch arbeiten $38 \%$ mit den mobilen Mini-PC.

RebekkaHöhl

\section{Office 365 für das iPhone}

Microsoft steigt behutsam in den Markt der Office-Anwendungen für das Apple-Betriebssystem iOS ein. In den USA brachte der Software-Konzern jetzt die App „Office Mobile for Office $365^{\circ}$ in den iTunes-Store von Apple, die derzeit aber nur für das iPhone optimiert ist, nicht für das iPad. Die kostenlose Anwendung kann in Verbindung mit einem Abo des Online-Pakets „Office 365“ genutzt werden und ermöglicht, Word-, Excel- und PowerPoint-Dokumente zu bearbeiten. Wann „Office Mobile für Office 365“ auch hierzulande angeboten wird, ist noch unklar. 